九州大学学術情報リポジトリ

Kyushu University Institutional Repository

\title{
On the Filter Problem of a Stationary Stochastic Process
}

Kano, Seigo

Kyushu University

https://doi.org/10.5109/12961

出版情報 : 統計数理研究. 5 (3/4)，pp.47-51，1953-06. Research Association of Statistical Sciences バージョン：

権利関係 : 


\title{
ON THE FILTER PROBLEM \\ OF A STATIONARY STOCHASTIC PROCESS ${ }^{(1)}$
}

\author{
By Seigo Kano
}

1. Introduction. In the process of gathering or transmitting information by mechanical or electrical means the signal that contains the information frequently becomes distorted. When this distortion has random statistical features it is called noise. In many applications it is necessary to remove the noise and recapture the original message. This process is called filtering. From the statistical view point, this filter problem has been treated by many authors, N. Wiener, Andre Blanc-Lapierre and Ulf. Grenander. But these works are mainly concerned with the signal in which the message and noise are linearly combined. In this paper we consider the statistical interaction effect between message and noise by means of $\mathrm{N}$. WIENER's theory.

2. Let $x(t)$ is a stationary stochastic process representing the message, $y(t)$ and $z(t)$ are two stationary stochastic processes representing the noise and denote the auto-correlation functions of these processes by $r_{x x}, r_{y y}$ and $\boldsymbol{r}_{z z}$ respectively. Further in this paper we make the following assumptions:

(1) mean functions of these processes are all zero,

(2) auto-correlation functions $r_{x x}, r_{y y}$ and $r_{z z}$ have the spectral density functions $\Phi_{x x}, \Phi_{y y}$ and $\Phi_{z z}$ respectively,

(3) $x(t)$ and $y(t)$ are stationarily correlated and the crosscorrelation function $r_{x y}$ is expressed as

$$
r_{x y}(t)=\frac{1}{2 \pi} \int_{-\infty}^{\infty} e^{i t \omega} \Phi_{x y}(\omega) d \omega
$$

(4) $z(t)$ is independent of $x(t)$ and $y(t)$.

Under these assumptions our main result is stated as following

Theorem 1. If $r(t)=r_{y y}(t)+r_{x x}(t) r_{z z}(t)$ is written as

$$
r(t)=\frac{1}{2 \pi} \int_{-\infty}^{\infty} e^{i t s} \phi(x) d x
$$

and

$$
\Phi(\omega)=|\Psi(\omega)|^{2},
$$

where $\phi(\omega)$ has no real zeros and $\phi(\omega)$ is retional function free from zeros and poles in the lower half-plane, then the $K(\sigma)$ which is of limited total variations and minimizes the error variance

(1) At the Autum Meeting of Japanese Math. Soc. at Kyoto Univ., in Nov. 3, 1952. 
(4) $E \mid x(t+\alpha)-\int_{0}^{\infty}\{y(t-\sigma)+x(t-\sigma) z(t-\sigma)\} d K(\sigma)^{2}$, is given by

$$
k(\omega)=\frac{1}{2 \pi \Psi(\omega)} \int_{0}^{\infty} e^{-i \omega t} d t \int_{-\infty}^{\infty} \frac{J_{x y}(u)}{\Psi(u)} e^{i u(t+\alpha)} d u,
$$

where we put

$$
k(\omega)=\int_{0}^{\infty} e^{-i \omega \sigma} d K(\sigma) .
$$

Proof. our error variance (4) is computed as follows

$$
\begin{aligned}
& E\left|x(t+\alpha)-\int_{0}^{\infty}\{y(t-\sigma)+x(t-\sigma) z(t-\sigma)\} d K(\sigma)\right|^{2} \\
& =r_{x x}(0)-2 R\left[\int_{0}^{\infty} r_{x y}(\alpha+\tau) d \overline{K(\tau)}\right] \\
& +\int_{0}^{\infty} d \overline{K(\tau)} \int_{0}^{\infty}\left\{r_{y y}(\tau-\sigma)+r_{x x}(\tau-\sigma) r_{z z}(\tau-\sigma)\right\} d K(\sigma) \\
& =r_{x x}(0)-2 R\left[\int_{0}^{\infty} r_{x y}(\alpha+\tau) d \overline{K(\tau)}\right] \\
& +\int_{0}^{\infty} d \overline{K(\tau)} \int_{0}^{\infty} r(\tau-\sigma) d K(\sigma) .
\end{aligned}
$$

Let us suppose that

$$
\boldsymbol{r}_{x y}(\alpha+\tau)=\int_{0}^{\infty} \boldsymbol{r}(\tau-\sigma) d Q(\sigma)
$$

then (7) becomes

$$
\begin{gathered}
\boldsymbol{r}_{x x}(0)+\int_{0}^{\infty} d[K(\sigma)-Q(\sigma)] \int_{0}^{\infty} d[\overline{K(\tau)}-\overline{Q(\tau)}] r(\tau-\sigma) \\
-\int_{0}^{\infty} d Q(\sigma) \int_{0}^{\infty} r(\tau-\sigma) d \overline{Q(\tau)}
\end{gathered}
$$

Let us now suppose that

$$
\begin{aligned}
& \int_{0}^{\infty} e^{-i \omega t} d K(t)=k(\omega) \\
& \int_{0}^{\infty} e^{-i \omega t} d Q(t)=q(\omega)
\end{aligned}
$$

then by (2) the expression ( 9 ) becomes

(12) $r_{x x}(0)-\frac{1}{2 \pi} \int_{-\infty}^{\infty}|q(\omega)|^{2} \Phi(\omega) d \omega+\frac{1}{2 \pi} \int_{-\infty}^{\infty} k(\omega)-\left.q(\omega)\right|^{2} \Phi(\omega) d \omega$, 
which will attain its minimum when

$$
q(\omega)=h(\omega)
$$

and its minimum will be

$$
r_{x x}(0)-\frac{1}{2 \pi} \int_{-\infty}^{\infty} \mathscr{U}(\omega) \mid q(\omega)^{2} d \omega .
$$

Accordingly it is sufficient to find a solution of the equation

$$
r_{x y}(\tau+\alpha)=\int_{0}^{\infty} r(\tau-\sigma) d K(\sigma)
$$

For this purpose we use the factorization of $\Phi(\omega)$, i.e. (3). So if we put

$$
\zeta^{\prime}(t)=\frac{1}{2:} \int_{-\infty}^{\infty} \bar{\Psi}((\prime)) e^{i \omega t} d \omega
$$

then

$$
\varsigma^{\prime}(t)=0 \quad t<0
$$

hence we can write

$$
\begin{aligned}
\boldsymbol{r}(\tau) & =\int_{0}^{\infty} \varphi(t+\tau) \overline{\varphi(t)} d t & & \tau>0 \\
& =\int_{-\tau}^{\infty} \varphi(t+\tau) \overline{\varphi(t)} d t & & \tau<0 .
\end{aligned}
$$

Then (15) becomes

Now let us put

$$
\begin{aligned}
\boldsymbol{r}_{x y}(\alpha+t)= & \int_{0}^{t} d \overline{K(\tau)} \int_{0}^{\infty} \varphi(t-\tau+\sigma) \overline{\psi(\sigma)} d \sigma \\
& +\int_{t}^{\infty} d \overline{K(\tau)} \int_{\tau-t}^{\infty} \varphi(t-\tau+\sigma) \overline{\psi(\sigma)} d \sigma \\
= & \int_{0}^{\infty} \overline{\xi^{\prime}(\sigma)} d \sigma \int_{0}^{\sigma+t} \varphi(t-\tau+\sigma) d \overline{K(\tau)} \quad t>0
\end{aligned}
$$

$$
r_{x y}(\alpha+t)=\int_{0}^{\infty} \overline{\xi^{\prime}(\sigma)} L(\sigma+t) d \sigma \quad(-\infty<t<\infty) .
$$

We see that if we extend ' $\varphi^{\prime}$ and $d K(\sigma)$ to cover negative arguments, for which $d K(\sigma)$ is to vanish, then

$$
\int_{-\infty}^{\infty} \xi(t-\tau) d K(\tau)=\left\{\begin{array}{cc}
L(t) & t>0 \\
0 & t<0 .
\end{array}\right.
$$

If we put

$$
\int_{-\infty}^{\infty} L(t) e^{-i \omega t} d t \equiv l(\omega)
$$

equation (20) becomes 


$$
\Phi_{x_{y}}(\omega) e^{i \omega \omega}=\bar{\Psi} \overline{(\omega)} l(\omega),
$$

which leads to

$$
l(\omega)=\frac{\Phi_{x y}(\omega)}{\Psi(\omega)} e^{i \omega a}
$$

or

$$
L(t)=\frac{1}{2 \pi} \int_{-\infty}^{\infty} \frac{\phi_{x y}(\omega) e^{i \omega \alpha}}{T(\omega)} e^{i \omega \alpha} d \omega .
$$

Now we substitutes (25) into (21) and make a formal transformation of both side, then this yields us

$$
\Psi(\omega) k(\omega)=\frac{1}{2 \pi} \int_{0}^{\infty} e^{-i \omega t} d t \int_{-\infty}^{\infty} \frac{\Phi_{x y}(u) e^{i u(t+\alpha)}}{\Psi(u)} d u
$$

or

$$
k(\omega)=\frac{1}{2 \pi \Psi(\omega)} \int_{0}^{\infty} e^{-i \omega t} d t \int_{-\infty}^{\infty} \frac{\mathscr{D}_{x y}(u)}{\Psi(u)} e^{i u(t+\alpha)} d u .
$$

The denominator of this expression is a rational function free from zeros in the lower half-plans, while the numerator is free from singularities in the lower half-plane. Thus $k(\omega)$ is of at most rational growth in the lower half-plane, in which it is free of singularities.

So that if

$$
k(\omega)=\int_{-\infty}^{\infty} e^{-i \omega t} d K_{1}(t)
$$

then $K(t)$ is constant for nagative arguments and is a possible $K(t)$. Thus our theorem was proved.

3. Example. Let us now simply illustrate our Theorem 1. We consider the following case.

$$
\begin{aligned}
& \boldsymbol{r}_{x x}(\tau)=\boldsymbol{r}_{y y}(\tau)=\boldsymbol{r}_{z z}(\tau)=e^{-\tau} \\
& \boldsymbol{r}_{s y}(\tau)=\varphi e^{-\tau} .
\end{aligned}
$$

Then

$$
\begin{aligned}
& V_{x x}(\omega)=\Phi_{y y}(\omega)=\Phi_{z z}(\omega)=\frac{1}{1+\omega^{2}}, \\
& \psi_{x y}(\omega)=\frac{\rho}{1+\omega^{2}}, \quad r(t)=e^{-|t|}+e^{-2 t i}, \\
& y(\omega)=\frac{5+2 \omega^{2}}{\left(1+\omega^{2}\right)\left(4+\omega^{2}\right)}, \Psi(\omega)=\frac{\left(\gamma^{2} \omega-\sqrt{5} i\right.}{(\omega-i)(\omega-2 i)} .
\end{aligned}
$$

Accordingly 


$$
\begin{aligned}
& \frac{1}{2 \pi} \int_{-\infty}^{\infty} \frac{\Phi_{x y t}(u)}{\Psi(u)} e^{i u\langle t+\infty)} d u=\frac{-e^{-(i+\infty)}}{v^{\prime} 5+\mu_{2}} i_{\rho} \\
& k(\omega)=\frac{(2 i-\omega) e^{-x}}{(\sqrt{2} \omega-\sqrt{5} i)(5+\sqrt{2})} \rho .
\end{aligned}
$$

Further the minimum error variance is given by

$$
E(\alpha)=1-\frac{1}{2} \cdot \frac{e^{-2 \alpha}}{\left(V^{\prime}+V^{2}\right)} .
$$

\section{References}

[1] N. Wiener : Extrapolation, interpolation and smoothing of stationary time series. (1949).

[2] Andre. Blanc-Lapierre: Sur certains fonctions aleatoires stationnaires. A la faculte des sciences de L'universite de Paris. (1945).

[3] Ulf. Grenander: Sorchastic process and statistical inference. Akiv for Math. 1. (1950). 